\title{
In vitro Antimicrobial Activity of Some Synthetic Indolium Chloride Derivatives
}

\author{
Arifa Akther ${ }^{1}$, Mohammad Rashedul Haque ${ }^{2}$, Mohammad A. Rashid ${ }^{2}$ and \\ Md. Wahab Khan ${ }^{1}$ \\ ${ }^{1}$ Department of Chemistry, Bangladesh University of Engineering and Technology \\ Dhaka-1000, Bangladesh \\ ${ }^{2}$ Department of Pharmaceutical Chemistry, University of Dhaka, Dhaka-1000, Bangladesh
}

(Received: September 22, 2020; Accepted: November 15, 2020; Published (web): December 10, 2020)

\begin{abstract}
A total of eight compounds including four starting materials and four indolium chloride derivatives were tested against Gram positive and Gram negative bacterial and fungal strains. Among them, $N$-acetyl-2acetylindoliumchloride (8) exhibited significant antimicrobial activity at a concentration of $200 \mu \mathrm{g} / \mathrm{disc}$, while the $\mathrm{N}$ acetyl-2-benzoylindoliumchloride (9) showed moderate activity at $400 \mu \mathrm{g} / \mathrm{disc}$. At the same time, $\mathrm{N}$-acetyl-2anisoylindoliumchloride (10) and $N$-acetyl-2-tolylindoliumchloride (11) demonstrated weak antimicrobial activity.
\end{abstract}

Key words: $N$-acetyl-2-acetylindoliumchloride, antimicrobial assay, disc diffusion.

\section{INTRODUCTION}

In many developed countries, most antibiotics are expensive and inaccessible for a significant percentage of the patients. Antibiotic resistance further compromises efficacy of the treatment. Many bacterial strains, including those that cause common infections of the skin, throat, urinary tract and lungs, are becoming immune to the widely available antibiotics leading to increased difficulty for treatment, misery and death. ${ }^{1-7}$ Therefore, the production of new synthetic antimicrobial agents is a demand for time.

One of the biggest useful resources of novel compounds with diverse biological activities is heterocyclic chemistry, primarily due to the unusual ability of the resulting compounds to imitate the peptide structure and to bind reversibly to proteins. ${ }^{8-}$

${ }^{11}$ In the drug discovery studies, indole derivatives are very useful heterocyclic compounds. They represent an influential class of molecules which play major roles in the biology of cells and are potential products that occur naturally. The use of indole derivatives as

Correspondence to: Md. Wahab Khan

E-mail: mwkhan@chem.buet.ac.bd

Dhaka Univ. J. Pharm. Sci. 19(2): 133-137, 2020 (December)

DOI: https://doi.org/10.3329/dujps.v19i2.50628 bioactive molecules against bacteria, cancer cells and various types of disorders in the human body has been becoming increasingly significant. ${ }^{12}$

In the current investigation, some derivatives of indolium chloride (8-11) (Figure 1) were screened for antimicrobial activity against some pathogenic microorganisms. The objective of the current study was both to investigate their impact on the pathogens tested and to identify the lead compounds with strong antimicrobial activity.

\section{MATERIALS AND METHODS}

General experimental procedures. Melting points were measured with a Gallenkamp (England) melting point apparatus in open capillary tubes. FTIR spectra were recorded on a Shimadzu FTIR spectrophotometer. The UV spectra were obtained with a Shimadzu visible spectrophotometer in dry ethanol. The ${ }^{1} \mathrm{H}-(400 \mathrm{MHz})$ and ${ }^{13} \mathrm{C}-(100 \mathrm{MHz})$ NMR spectra were acquired on a Bruker DPX-400 spectrophotometer and the chemical shifts are reported with respect to the signal of the internal standard, tetramethylsilane (TMS). Column chromatography and TLC were conducted over silica gel (60-120 mesh) and pre-coated silica gel $60 \mathrm{~F}_{254}$ (E. 
Merck), respectively and the spots on TLC plates were visualized with UV light. On a Perkin-Elmer 240C Analyser, elemental analyses $(\mathrm{C}, \mathrm{H}, \mathrm{N})$ were performed. Acrylic esters, $\left(\mathrm{Ph}_{3} \mathrm{P}\right)_{2} \mathrm{PdCl}_{2}$, and other reagents were procured from Fluka (Switzerland) and E. Merck (Germany), respectively.<smiles>CC(=O)c1cc2ccccc2n1C(C)=O</smiles>

8

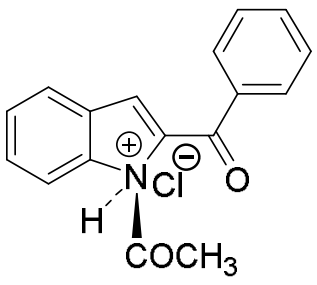

9
Synthesis of indolium chloride derivatives. The compounds used in the current study were synthesized as stated by the methods shown in Scheme

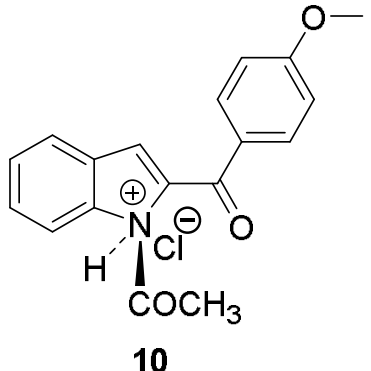

10

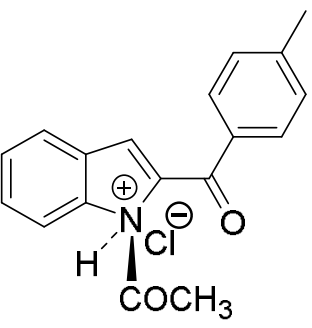

11

Figure 1. The synthesised indolium chloride derivatives.<smiles>CC(=O)Nc1ccccc1I</smiles>

1

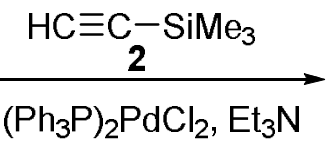
Cul, DMF $25^{\circ} \mathrm{C}, 24 \mathrm{~h}$

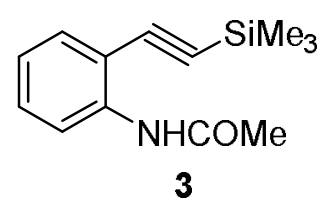<smiles></smiles>

8-11

$$
\begin{aligned}
& 4,8 \mathrm{R}=\mathrm{Me} \\
& 5,9 \mathrm{R}=\mathrm{C}_{6} \mathrm{H}_{5} \\
& 6,10 \mathrm{R}=\mathrm{C}_{6} \mathrm{H}_{4} \mathrm{OMe}-p \\
& 7,11 \mathrm{R}=\mathrm{C}_{6} \mathrm{H}_{4} \mathrm{Me}-p
\end{aligned}
$$

Scheme 1. Synthesis of indolium chloride derivatives.

Synthesis of $\mathrm{N}$-acetyl-2-substituted indolium chloride (8-11). N-acetyl-2-substituted indolium chloride (8-11) were prepared from 2-iodoacetanilide through palladium-catalysed reaction using trimethylsilyl acetylene followed by Friedel-Craft acylation of the resulting 2-(trimethylsilyl)ethnylacetanilide with acyl chloride or acetic anhydride as shown in Scheme 1.

2-Iodoacetanilide (1) was easily reacted with trimethylsilyl acetylene (2) in $\quad \mathrm{N}, \mathrm{N}$-dimethylformamide (DMF) in presence of bis(triphenylphosphine) palladium (II) chloride (3.5 mol percent), trimethylamine (4 equiv), and copper(I) iodide ( $8 \mathrm{~mol}$ percent) at room temperature for 24 hours under the nitrogen atmosphere, giving 2-(trimethylsilyl)ethynylacetanilide (3). The latter acetanilide was then acylated at $0-25{ }^{\circ} \mathrm{C}$ for 2 hours by Friedel-Craft reaction, stirring a mixture of $\mathbf{3}$, anhydrous $\mathrm{AlCl}_{3}$ (3 equiv) and acyl chloride (1 quiv) in tetrachloroethane to yield 2-acyl indole derivatives 8-11.

2-Iodoacetanilide (1) was synthesised from 2iodoanilines by acetylation reaction for 3 hours with $\left(\mathrm{CH}_{3} \mathrm{CO}\right)_{2} \mathrm{O} / \mathrm{CH}_{3} \mathrm{COOH} / \mathrm{Zn}$ at $80{ }^{\circ} \mathrm{C}$ and 2iodoanilines was prepared from aniline using $\mathrm{KI} / \mathrm{KIO}_{3} / \mathrm{H}^{+}$in methanol by following a published method. ${ }^{13}$

2-Iodoacetanilide (1). White crystalline solid, mp 108-109 ${ }^{\circ} \mathrm{C}$; $\mathrm{R}_{\mathrm{f}}$ value 0.36 (n-hexane); IR (KBr): $v_{\max } 3273.0(\mathrm{NH}), 1660.6(\mathrm{C}=\mathrm{O}), 1573.8,1529.4$, 1463.9, 14433.0, 1411.8, 1292.2, 1253.6, 1014.5, $750.3,663.5 \mathrm{~cm}^{-1} ;{ }^{1} \mathrm{H}$ NMR $\left(400 \mathrm{MHz}, \mathrm{CDCl}_{3}\right): \delta$ 8.15 (s, 1H, Ar-H), 7.75 (d, 1H, $J=8.0 \mathrm{~Hz}, \operatorname{Ar}-\mathrm{H})$, 7.42 (br. s, 1H, NH), 7.31 ( t, $1 \mathrm{H}, J=8 \mathrm{~Hz}, \mathrm{Ar}-\mathrm{H}$ ), $6.82(\mathrm{t}, 1 \mathrm{H}, J=8.0 \mathrm{~Hz}, \mathrm{Ar}-\mathrm{H}), 2.20\left(\mathrm{~s}, 3 \mathrm{H}, \mathrm{COCH}_{3}\right)$. 
2-(Trimethylsilyl)ethynylacetanilides(3). Crystalline solid; $\mathrm{mp} 94-95{ }^{\circ} \mathrm{C}$; UV (EtOH): $\lambda_{\max }=296.2$, $250.8 \mathrm{~nm}$; IR (KBr): $v_{\max } 3327(\mathrm{NH}), 2158(\mathrm{C} \equiv \mathrm{C})$, $1695(\mathrm{C}=\mathrm{O}), 1672,1576,1516,1444 \mathrm{~cm}^{-1}$; ${ }^{1} \mathrm{H}$ NMR (400 MHz CDCl 3 ): $\delta 8.39$ (d, $J=8.3 \mathrm{~Hz}, 1 \mathrm{H}, \mathrm{Ar}-\mathrm{H})$, 7.99 (br.s, 1H, NH), 7.41 (dd, $J=8.0,8.3 \mathrm{~Hz}, 1 \mathrm{H}$, Ar-H), 7.32 (dd, $J=8.0,8.3 \mathrm{~Hz}, 1 \mathrm{H}$, Ar-H), 7.01 (d, $J=8.0 \mathrm{~Hz}, 1 \mathrm{H}, \mathrm{Ar}-\mathrm{H}), 2.21\left(\mathrm{~s}, 3 \mathrm{H}, \mathrm{COCH}_{3}\right), 0.30$ (s, 9H, $\left.\mathrm{SiMe}_{3}\right) ;{ }^{13} \mathrm{C}$ NMR $\left(100 \mathrm{MHz}, \mathrm{CDCl}_{3}\right): \delta 168.0$ (CO), 139.5, 131.4, 129.9, 123.10, 118.9, 111.5, 102.2, 100.2, $24.7\left(\mathrm{CH}_{3}\right), 0.10\left(\mathrm{SiMe}_{3}\right)$; Anal. calcd. for $\mathrm{C}_{13} \mathrm{H}_{17} \mathrm{SiNO}$ : $\mathrm{C}, 67.48 ; \mathrm{H}, 7.40 ; \mathrm{N}, 6.05$. Found: C, 67.09; H, 7.43; N, 6.03.

N-Acetyl-2-acetyl indolium chloride (8). Crystalline solid, mp $84-86{ }^{\circ} \mathrm{C}$; UV (EtOH): $\lambda_{\max } 334$, 305.4, 259.8, $238.4 \mathrm{~nm}$; IR (KBr): $v_{\max } 3221(\mathrm{NH})$, $1684(\mathrm{C}=\mathrm{O}), 1652,1608,1576,1560,1502,1130 \mathrm{~cm}^{-}$ ${ }^{1}$; ${ }^{1} \mathrm{H}$ NMR (400 MHz $\mathrm{CDCl}_{3}$ ): $\delta 11.06$ (br.s, $1 \mathrm{H}$, $\mathrm{NH}), 8.59$ (d, 1H, $J=8.0 \mathrm{~Hz}$, Ar-H), 7.65 (t, 1H, $J=$ $8.0 \mathrm{~Hz}, \mathrm{Ar}-\mathrm{H}), 7.46$ (d, 1H, J = 8.0 Hz, Ar-H), 7.05 (t, $1 \mathrm{H}, J=8.0 \mathrm{~Hz}, \mathrm{Ar}-\mathrm{H}), 6.10$ (s, 1H, H-3), 2.18 (s, $\left.3 \mathrm{H}, \mathrm{COCH}_{3}\right), 2.12\left(\mathrm{~s}, 3 \mathrm{H}, \mathrm{COCH}_{3}\right) ;{ }^{13} \mathrm{C}$ NMR $(100$ $\left.\mathrm{MHz}, \mathrm{CDCl}_{3}\right): \delta 194.31(\mathrm{C}=\mathrm{O}), 185.31(\mathrm{C}=\mathrm{O})$, $168.89,140.152,135.85,133.83,129.06,122.41$, $121.02,98.02,25.40\left(\mathrm{COCH}_{3}\right), 23.15\left(\mathrm{COCH}_{3}\right)$.

$\mathrm{N}$-Acetyl-2-benzoyl-1H-indolium chloride (9). White crystalline solid, mp $63-64{ }^{\circ} \mathrm{C}$; IR (KBr): $v_{\max }$ $3274.9(\mathrm{NH}), 3076.2(\mathrm{C}-\mathrm{H}), 1703.0(\mathrm{C}=\mathrm{O}), 1575.7$, $1529.7(\mathrm{C}=\mathrm{C})$ and 1463.9, 1380.9, $1276.8 \mathrm{~cm}^{-1} ;{ }^{1} \mathrm{H}$ NMR (400 MHz, $\mathrm{CDCl}_{3}$ ): $\delta 11.08$ (br. s, $1 \mathrm{H}, \mathrm{NH}$ ), $8.64(\mathrm{~d}, 1 \mathrm{H}, J=8.4 \mathrm{~Hz}, \mathrm{Ar}-\mathrm{H}), 7.94(\mathrm{~d}, 2 \mathrm{H}, J=7.2$ $\mathrm{Hz}, \mathrm{Ar}-\mathrm{H})), 7.82(\mathrm{~d}, 1 \mathrm{H}, J=8.0 \mathrm{~Hz}, \mathrm{Ar}-\mathrm{H}) 7.52(\mathrm{~m}$, 4H, Ar-H), 7.13 (t, 1H, Ar-H), 6.79 (s, 1H, H-3), 2.23 (s, 3H, $\left.\mathrm{COCH}_{3}\right) ;{ }^{13} \mathrm{C}$ NMR $\left(100 \mathrm{MHz}, \mathrm{CDCl}_{3}\right): \delta$ $194.70(\mathrm{C}=\mathrm{O}), \quad 179.53(\mathrm{C}=\mathrm{O}), 168.98, \quad 140.11$, $133.99,133.84,132.58,129.15,128.82,126.91$, $123.12,122.79,121.51,94.99,25.49\left(\mathrm{COCH}_{3}\right)$.

$\mathrm{N}$-Acetyl-2-anisoyl indolium chloride (10). Light yellow crystal, mp. $112-113{ }^{\circ} \mathrm{C}$; UV (EtOH): $\lambda_{\max } 380.80,360.6,238.0 \mathrm{~nm}$; IR (KBr): $v_{\max } 3327.9$ $(\mathrm{NH}), 1683.7(\mathrm{C}=\mathrm{O}), 1610,1576.7,1500,1413$, 1360, $1175 \mathrm{~cm}^{-1} ;{ }^{1} \mathrm{H}$ NMR (400 MHz, $\mathrm{CDCl}_{3}$ ): $\delta$ 11.04 (br. s, $1 \mathrm{H}, \mathrm{NH}), 8.62$ (d, 1H, $J=8.0 \mathrm{~Hz}, \mathrm{Ar}-\mathrm{H}$ ), 7.91 (d, 2H, $J=8.0 \mathrm{~Hz}$, Ar-H), 7.77 (d, 1H, $J=8.0$
$\mathrm{Hz}, \mathrm{Ar}-\mathrm{H}), 7.51(\mathrm{t}, 1 \mathrm{H}, J=7.2 \mathrm{~Hz}, \mathrm{Ar}-\mathrm{H}), 7.12$ (d, $1 \mathrm{H}, J=7.2 \mathrm{~Hz}, \mathrm{Ar}-\mathrm{H}), 6.97$ (d, 2H, $J=8 \mathrm{~Hz}, \mathrm{Ar}-\mathrm{H})$, $6.70(\mathrm{~s}, 1 \mathrm{H}, \mathrm{H}-3), 3.88$ (s, 3H, Ar- $\left.-\mathrm{OCH}_{3}\right), 2.22$ (s, $\left.3 \mathrm{H}, \mathrm{COCH}_{3}\right) ;{ }^{13} \mathrm{C}$ NMR $\left(100 \mathrm{MHz}, \mathrm{CDCl}_{3}\right): \delta 193.46$ $(\mathrm{C}=\mathrm{O}), 180(\mathrm{C}=\mathrm{O}), 168.93,163.41,139.83,133.60$, $131.17,128.99,126.14,123.38,122.75,121.46$, 114.09, 94.00, $55.53\left(\mathrm{OCH}_{3}\right) 25.48\left(\mathrm{COCH}_{3}\right)$. Anal. calcd for $\mathrm{C}_{18} \mathrm{H}_{16} \mathrm{ClNO}_{3}: \mathrm{C}, 65.56 ; \mathrm{H}, 4.89 ; \mathrm{N}, 4.25$. Found: C, 65.87; H, 4.75; N, 4.52.

$\mathrm{N}$-Acetyl-2-toluoyl indolium chloride (11). Amorphous solid, mp. $64-65{ }^{\circ} \mathrm{C}$. UV (EtOH): $\lambda_{\max }$ 361.4, $256.6 \mathrm{~nm}$; IR (KBr): $v_{\max } 3325(\mathrm{NH})$, 1687(C=O), 1569, 1508, 1446, 1423, 1365, 1190, $756 \mathrm{~cm}^{-1} ;{ }^{1} \mathrm{H}$ NMR $\left(400 \mathrm{MHz}, \mathrm{CDCl}_{3}\right): \delta 11.07$ (br. s, $1 \mathrm{H}, \mathrm{NH}), 8.63(\mathrm{~d}, 1 \mathrm{H}, J=8.0 \mathrm{~Hz}, \mathrm{Ar}-\mathrm{H}), 7.83(\mathrm{~d}, 2 \mathrm{H}$, $J=8.0 \mathrm{~Hz}, \mathrm{Ar}-\mathrm{H}), 7.78(\mathrm{~d}, 1 \mathrm{H}, J=8.0 \mathrm{~Hz}, \mathrm{Ar}-\mathrm{H})$, 7.49 (t, $1 \mathrm{H}, J=7.2 \mathrm{~Hz}$, Ar-H), 7.27 (d, 2H, $J=7.2$, Ar-H), 7.11 (t, 1H, J= 8.0 Hz, Ar-H), 6.74 (s, 1H, H3), $2.41\left(\mathrm{~s}, 3 \mathrm{H}, \mathrm{COCH}_{3}\right), 2.21\left(\mathrm{~s}, 3 \mathrm{H}, \mathrm{Ar}-\mathrm{CH}_{3}\right) ;{ }^{13} \mathrm{C}$ NMR $\left(100 \mathrm{MHz}, \mathrm{CDCl}_{3}\right): \delta 198.92(\mathrm{C}=\mathrm{O}), 179.85$ $(\mathrm{C}=\mathrm{O}), 168.88,143.45,139.97,133.74,131.71$, $128.83,126.16,123.16,122.68,121.12,120.81$, 94.48, $25.48\left(\mathrm{COCH}_{3}\right), 21.62\left(\mathrm{Ar}-\mathrm{CH}_{3}\right)$. Anal. calcd for $\mathrm{C}_{18} \mathrm{H}_{16} \mathrm{ClNO}_{2}$ : C, 68.90; H, 5.14; N, 4.46. Found: C, 69.20; H, 5.40; N, 4.77.

Antimicrobial screening. Disc diffusion method $^{14}$ was used to assess the antimicrobial activity of the test compounds, and the results observed are listed in Table 1. The samples were dissolved in chloroform/methanol at a ratio of 1:1 separately and applied at the concentration of $200-$ and $400-\mu \mathrm{g} / \mathrm{disc}$ to sterile filter paper disks. For antibacterial and antifungal assay kanamycin (30 $\mu \mathrm{g} / \mathrm{disc})$ and griseofulvin $(25 \mu \mathrm{g} / \mathrm{disc})$, respectively were used as positive controls. The standard test microorganisms were collected from the Institute of Nutrition and Food Sciences (INFS), University of Dhaka, Bangladesh.

\section{RESULTS AND DISCUSSION}

For antimicrobial activity screening a total of four $\mathrm{N}$-acetyl-2-substituted indolium chloride (8-11) have been tested against five Gram positive, eight Gram negative bacteria and three fungi. It is clear 
from the antimicrobial screening (Table 1) that very prominent antimicrobial activity was revealed by $\mathrm{N}$ acetyl-2-acetylindoliumchloride (8). $\mathrm{N}$-acetyl-2benzoylindoliumchloride (9), on the other hand, demonstrated mild to moderate activity, while $\mathrm{N}$ acetyl-2-anisoylindoliumchloride (10) and $\mathrm{N}$-acetyl2-tolylindoliumchloride (11) displayed mild inhibition of microbial growth.

Table 1. Antimicrobial activity of compounds 8-11.

\begin{tabular}{|c|c|c|c|c|c|c|}
\hline \multirow[t]{3}{*}{ Test microorganisms } & \multicolumn{6}{|c|}{ Diameter of zone of inhibition $(\mathrm{mm})$} \\
\hline & \multicolumn{2}{|c|}{8} & 9 & 10 & 11 & \multirow{2}{*}{$\begin{array}{l}\text { Kanamycin }(30 \mu \mathrm{g} / \mathrm{disc}) / \\
\text { Griseofulvin }(25 \mu \mathrm{g} / \mathrm{disc})\end{array}$} \\
\hline & \multicolumn{5}{|c|}{$200 \mu \mathrm{g} / \mathrm{disc} 400 \mu \mathrm{g} / \mathrm{disc} 400 \mu \mathrm{g} / \mathrm{disc} 400 \mu \mathrm{g} / \mathrm{disc} 400 \mu \mathrm{g} / \mathrm{disc}$} & \\
\hline \multicolumn{7}{|l|}{ Gram positive bacteria } \\
\hline Bacillus cereus (BTCC-19) & 16.4 & 18.2 & 10.5 & 9.4 & 8.6 & 35.1 \\
\hline B. megaterium (BTCC-18) & 21.9 & 22.0 & 13.2 & 8.9 & 9.9 & 37.8 \\
\hline B. subtilis (QL 40) & 14.4 & 14.5 & 9.9 & 8.4 & 8.3 & 33.4 \\
\hline Staphylococcus aureus (BTCC-43) & 15.4 & 17.0 & 11.4 & 9.6 & 9.1 & 36.1 \\
\hline Sarcina lutea (ATCC-9341) & 15.7 & 21.0 & 11.9 & 7.4 & 8.7 & 32.2 \\
\hline \multicolumn{7}{|l|}{ Gram negative bacteria } \\
\hline Salmonella paratyphi (AM 16590) & 16.1 & 14.5 & 12.4 & 9.9 & 10.2 & 32.9 \\
\hline S. typhi (AM 16406) & 16.2 & 30.1 & 11.9 & 9.6 & 9.7 & 33.6 \\
\hline Vibrio parahemolyticus (AM 16362) & 18.1 & 21.0 & 12.3 & 9.8 & 9.7 & 37.5 \\
\hline V. mimicus (N 1967) & 14.5 & 17.0 & 12.5 & 9.5 & 10.2 & 36.9 \\
\hline $\begin{array}{l}\text { Escherichia coli } \\
\text { (BTCC-172) }\end{array}$ & 14.8 & 20.0 & 9.9 & 8.7 & 9.4 & 34.7 \\
\hline Shigella dysenteriae (ATCC 26131) & 17.4 & 16.5 & 11.8 & 9.2 & 9.8 & 36.3 \\
\hline S. boydii (ATCC 13147) & 15.7 & 21.6 & 11.9 & 7.4 & 8.7 & 35.5 \\
\hline Pseudomonas aeruginosa & 14.2 & 19.5 & 10.1 & 8.4 & 9.2 & 36.9 \\
\hline \multicolumn{7}{|l|}{ Fungi } \\
\hline Saccharomyces cerevisiae & 13.7 & 17.0 & 11.5 & 8.8 & 9.4 & 31.3 \\
\hline Candida albicans & 17.7 & 17.0 & 11.7 & 9.6 & 10.1 & 37.2 \\
\hline Aspergillus niger & 16.9 & 20.0 & 12.4 & 8.6 & 9.8 & 33.3 \\
\hline
\end{tabular}

Diffusion time- 23 hours; Solvent used to dissolve the samples- $\mathrm{CHCl}_{3} / \mathrm{MeOH}-1: 1$

In the screening, compound $\mathbf{8}$ exhibited maximum zone of inhibition. This compound was tested for twice at the concentration of 200- and 400$\mu \mathrm{g} / \mathrm{disc}$ and the activity in term of zone of inhibition was compared with the standard drugs, kanamycin and griseofulvin. Among the five Gram positive bacteria, the antimicrobial activity of compound $\mathbf{8}$ against $S$. lutea was highest with mean zone of inhibition of $21 \mathrm{~mm}$ and for Gram negative bacteria and the highest zone of inhibition was found for $S$. typhi $(30 \mathrm{~mm})$. In case of antifungal activity, A. niger was found to be most sensitive $(20 \mathrm{~mm})$ to the test compound $\mathbf{8}$ than the other fungal strains.
Compound 9 revealed the second-highest antimicrobial activity. It exhibited significant activity against $B$. megaterium, $V$. parahemolyticus, $V$. mimicus and $A$. niger. During antimicrobial screening against 16 bacteria and 3 fungi, compounds $\mathbf{1 0}$ and 11 displayed weak inhibitory zone ranging from 7.4 to $10.2 \mathrm{~mm}$, where they showed maximum activity against $S$. paratyphi and $V$. mimicus, respectively. The antimicrobial activity of intermediate $\mathbf{3}$ has also been screened but it revealed no activity.

\section{CONFLICT OF INTEREST}

No conflict of interest is declared by the authors. 


\section{ACKNOWLEDGEMENTS}

The research work was conducted by the financial support from Bangladesh University of Engineering and Technology (BUET), Dhaka, Bangladesh, the authors would like to thank the Department of Pharmaceutical Chemistry, Dhaka University, Bangladesh, for providing laboratory facility to conduct the antimicrobial screening of the tested compounds.

\section{REFERENCES}

1. Kunin, C.M. 1983. Antibiotic resistance - a world health problem we cannot ignore (Editorial). Ann. Intern. Med. 99, 859-860.

2. Kunin, C.M. 1993. Resistance to antimicrobial drugs - a worldwide calamity. Ann. Intern. Med. 118, 557-561.

3. Kunin, C.M., Lipton, H.L., Tupasi, T., Sacks, T., Scheckler, W.E. and Jivani, A. 1987. Social, behavioural, and practical factors affecting antibiotic use worldwide: report of Task Force 4. Rev. Infect. Dis. 9, 270-285.

4. Burke, J.P. and Levy, S.B. 1985. Summary report of worldwide antibiotic resistance: international task forces on antibiotic use. Rev. Infect. Dis. 7, 560-564.

5. Cohen, M.L. 1992. Epidemiology of drug resistance: implications for a post-antimicrobial era. Science, 257, 10501055 .
6. Neu, H.C. 1992. The crisis in antibiotic resistance. Science 257, 1064-1073.

7. Control of antibiotic-resistant bacteria: memorandum from a WHO meeting. 1983. Bull. World Health Organ. 61, $423-$ 433.

8. Dolle, R.E. 2001. Comprehensive survey of combinatorial library synthesis: 2000. J. Comb. Chem. 3, 477-517.

9. Dolle, R.E. and Nelson, Jr K.H. 1999. Comprehensive survey of combinatorial library synthesis: 1998. J. Comb. Chem. 1, 235-282.

10. Franzen, R.G. 2000. Recent advances in the preparation of heterocycles on solid support: A review of the literature. $J$. Comb. Chem. 2, 195-214.

11. Hanessian, S., McNaughton-Smith, G., Lombart, H.G. and Lubell, W.D. 1997. Design and synthesis of conformationally constrained amino acids as versatile scaffolds and peptide mimetics. Tetrahedron, 53, 12789-12854.

12. Kaushik, N.K., Kaushik, N., Attri, P., Kumar, N., Kim, C.H., Verma, A.K., Choi, E., 2013. Biomedical Importance of Indoles. Molecules, 18, 6620-6662.

13. Vogel, A.I. and Furniss, B.S. 1989. Vogel's Text book of Practical Organic Chemistry, 5th edn. Longman group: UK, p. 930 .

14. Bauer, A.W., Kirby, W.M.M., Sherries, J.C. and Truck, M. 1996. Antibiotic susceptibility testing by standard single disc diffusion method. Am. J. Clin. Pathol. 45, 426-493. 